\title{
Incidence and outcome of bone metastatic disease at University Malaya Medical Centre
}

\author{
Vivek Ajit Singh ${ }^{1}$, MS Ortho, FRCSE, Amber Haseeb ${ }^{1}$, MBBS, Alla Allden H Ali Alkubaisi ${ }^{1}$, MS Ortho
}

INTRODUCTION Morbidity and mortality from malignant diseases are usually the result of metastasis. The bone is the third most common site of metastasis.

METHODS This is a retrospective study of patients with metastatic bone disease who were referred to the Orthopaedic Department of University Malaya Medical Centre, Malaysia, between January 2004 and October 2009.

RESULTS A total of 151 patients ( $51.0 \%$ men, $49.0 \%$ women) had metastatic bone disease, with the highest incidence at the age range of 50-59 years. The commonest primary cancer was breast (23.3\%), followed by lung $(21.2 \%)$, prostate (9.3\%), thyroid $(7.3 \%)$ and renal cell carcinoma (5.3\%); unknown primary cancer was $6.6 \%$. There was long bone involvement in $52.7 \%$ of cases, axial bone in $44.5 \%$, and both long and axial bones in $2.8 \%$. The majority (90.1\%) were symptomatic, with pain as the commonest symptom. 106 (70.2\%) patients had pathological fractures. Neurological deficit was reported in $90.7 \%$ of patients, with $41.1 \%$ having extraskeletal metastases. $67.8 \%$ of the lesions were osteolytic, $24.3 \%$ were sclerotic, and $7.9 \%$, mixed. Palliative and therapeutic interventions were undertaken for $62.0 \%$ of patients. The mean survival times were: breast 21.0; thyroid 20.7; prostate 20.3; lung 16.0; and unknown primary cancer 32.6 months. CONCLUSION In our study, breast and lung cancers were the commonest primary cancers in metastatic bone disease. Most patients had more than one site of involvement, pain at presentation and pathological fractures. Surgery is beneficial to relieve pain and improve function and neurology. Duration of survival depends on the type of primary cancer and whether systemic metastasis is present.

Keywords: bone, metastasis, outcome, survival

\section{INTRODUCTION}

Most of the morbidity and mortality that arise from malignant disease is due to metastasis. The commonest site of metastasis is the lung, followed by the liver and bone. Bone metastasis is present in almost all cancers; it presents most frequently in prostate, lung and breast cancers. Metastasis to the bone also occurs in other cancer types, including carcinomas of the melanoma, kidney and bladder.(1) The presence of metastatic bone disease can dramatically affect a patient's quality of life due to increased pain, impaired mobility, increased incidence of fractures and spinal cord compression. Patients with advanced breast and prostate cancers would eventually develop bone metastases as their disease progresses. In breast and prostate cancers, the symptoms related to metastasis are frequently the first indication of disease. ${ }^{(2)}$ It is, however, difficult to quantify the prevalence of bone metastases, as the incidence is affected by the sensitivity of diagnostic tools and the duration of patient survival. Bone pain is highly suggestive of bone metastasis in patients with cancer. Some patients may present with pathological fractures and their associated complications (e.g. neurological impairments in spine metastasis). The spine is infamously known to be the most common site of skeletal metastasis. ${ }^{(3)}$

\section{METHODS}

We conducted a retrospective review of cases of metastatic bone disease in University Malaya Medical Centre (UMMC), a tertiary referral centre in Malaysia, over a five-year period. All patients who were referred to the Orthopaedic Oncology Unit of UMMC for bone metastasis from 1 January 2004 to 31 October 2009 were included in the present study. Data was obtained from the medical records of UMMC, and when necessary, patient survival data was obtained via telephone calls to the patients, their family members, or from the National Registration Department of Malaysia. The aim of the present study was to create a local profile of patients with bone metastatic disease, with regard to the disease epidemiology, as well as to study the outcomes and results of various forms of management of the disease. We also aimed to determine the survival time of the different types of metastatic bone cancer seen at UMMC.

Patients who had (a) a histologically proven primary tumour that was present with metastasis; (b) an unknown primary cancer with histologically proven bone metastasis; or (c) known primary cancer and radiologically proven bone metastasis were included in the study. Patients with primary bone cancers (e.g. osteosarcoma, Ewing's sarcoma and chondrosarcoma) and insufficient data in their medical records were excluded.

Data collected included patient demographics (e.g. patient's gender, ethnicity and age at presentation) and clinical data (e.g. symptoms at presentation, type of cancer, site of metastasis, functional status, complications). Patients who presented with long bone metastasis and fracture were assessed using the Mirels scoring system, ${ }^{(4)}$ to determine the relationship between Mirels score and the incidence of fracture. Survivorship was tested using Kaplan-Meier and P Weirs statistical analyses. The end point used 
to determine the survival rate was death. All statistical analyses were performed using the IBM Statistical Package for the Social Sciences software version 21 (IBM Corp, Armonk, NY, USA).

\section{RESULTS}

From 1 January 2004 to 31 October 2009, a total of 204 patients were diagnosed with metastatic bone disease and referred to the Orthopaedic Oncology Unit of UMMC. Of these 204 patients, only 151 patients who had complete records were included in the present study. A majority of the patients were of Chinese ethnicity $(51.0 \%)$, followed by Malay $(30.0 \%)$, Indian $(18.0 \%)$ and other races $(1.0 \%)$. This is representative of the demographics of all the patients who presented to UMMC during that time period. The gender distribution of the 151 patients was almost equal ( $51 \%$ men, $49 \%$ women). The mean age of the patients was 68 years. Most of the patients were aged 40-79 years, and the highest incidence of metastatic bone disease occurred in patients aged 50-59 years (Fig. 1). The youngest was an 18-year-old male patient with metastatic anaplastic meningioma.

Breast cancer $(23.2 \%)$ was the most common primary cancer, followed by lung $(21.2 \%)$, prostate $(9.3 \%)$ and thyroid $(7.3 \%)$ cancers, and renal cell carcinoma (5.3\%). The incidence of primary tumours of unknown origin was an infinitesimal $6.6 \%$ (Table I).

We observed a male predominance in lung cancer $(n=21$, $65.6 \%)$, nasopharyngeal carcinoma $(n=4,57.1 \%)$, renal cell carcinoma $(n=6,75.0 \%)$, oesophageal cancer $(n=1,100.0 \%)$, colorectal cancer $(n=4,66.7 \%)$, malignant melanoma $(n=1$, $100.0 \%)$, thyroid cancer $(n=5,45.5 \%)$ and multiple myeloma $(\mathrm{n}=3,75.0 \%)$. A female predominance was observed in tongue cancer $(n=1,100.0 \%)$, squamous cell carcinoma $(n=1,100.0 \%)$ and anaplastic meningioma $(n=1,100.0 \%)$.

More patients presented with long bone metastases than axial bone metastases. Of the 151 patients, 65 (43.0\%) presented with isolated long bone metastasis, 61 (40.4\%) with axial bone metastases and 25 (16.6\%) with both long and axial bone metastases. Most of the patients received a combination of treatments for metastatic bone disease. The treatment typically consisted of surgery, chemotherapy, radiotherapy and hormonal therapy. Surgery performed to treat the primary tumour (e.g. mastectomy and thyroidectomy) was not considered part of the treatment for metastatic bone disease. The orthopaedic team performed surgical fixation for therapeutic and palliative reasons in $62.0 \%$ of patients. Surgical procedures included decompression and stabilisation procedures for spinal metastasis, curettage and cementation for long bone metastasis, and long stem total hip replacement surgery for proximal femur metastasis.

Pain at presentation and post treatment was recorded. At the time of presentation, a majority of the patients (90.1\%) had symptomatic pain. Bone metastasis was an incidental finding in $9.9 \%$ of the patients. In our study cohort, pain of variable severity was the commonest symptom of metastatic bone disease. The severity of pain at presentation was graded according to the American Cancer Society Pain Severity Scale..$^{(5)}$ Of the 151 patients, $32.9 \%$ had mild pain at presentation, while

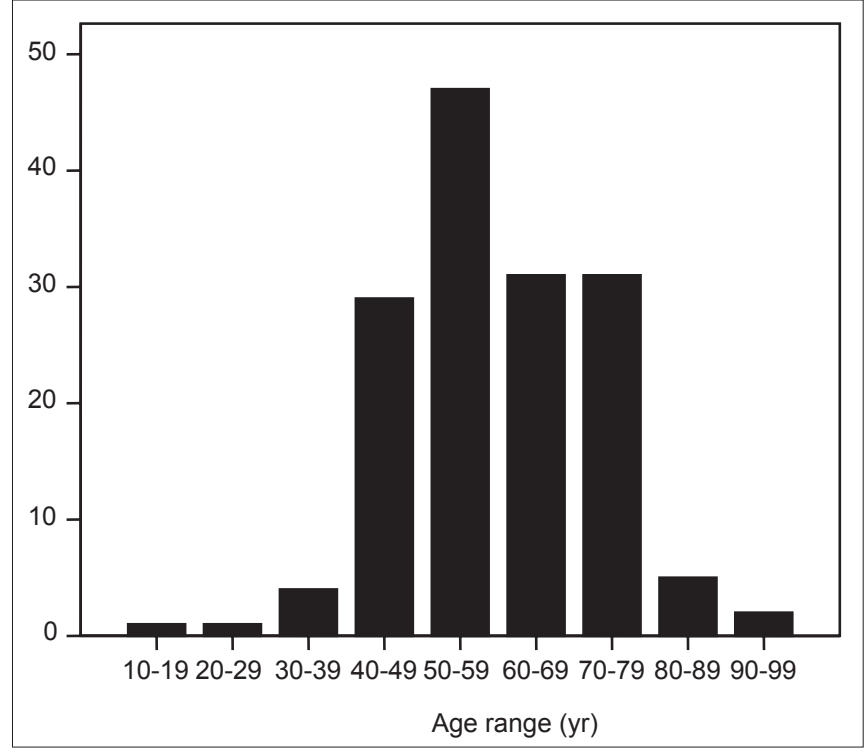

Fig. 1 Bar chart shows the age distribution of the study cohort $(n=151)$.

Table I. Types of primary cancers in the study cohort $(n=151)$.

\begin{tabular}{lc}
\hline Type of primary cancer & No. of patients (\%) \\
\hline Breast cancer & $35(23.2)$ \\
Lung cancer & $32(21.2)$ \\
Prostate cancer & $14(9.3)$ \\
Thyroid cancer & $11(7.3)$ \\
Renal cell carcinoma & $8(5.3)$ \\
Nasopharyngeal carcinoma & $7(4.6)$ \\
Colorectal cancer & $6(4.0)$ \\
Cervical cancer & $5(3.3)$ \\
Multiple myeloma & $4(2.6)$ \\
Hepatocellular carcinoma & $3(2.0)$ \\
Lymphoma & $3(2.0)$ \\
Testicular cancer & $3(2.0)$ \\
Carcinoma of the larynx & $1(0.7)$ \\
Ovarian cancer & $1(0.7)$ \\
Oesophageal cancer & $1(0.7)$ \\
Tongue cancer & $1(0.7)$ \\
Squamous cell carcinoma & $1(0.7)$ \\
Parathyroid cancer & $1(0.7)$ \\
Endometrial cancer & $1(0.7)$ \\
Anaplastic meningioma & $1(0.7)$ \\
Malignant melanoma & $1(0.7)$ \\
Basal cell carcinoma & $1(0.7)$ \\
\hline & $10(6.6)$ \\
\hline Nnknown &
\end{tabular}

$33.6 \%$ and $23.2 \%$ had moderate and severe pain, respectively. The severity of pain improved after treatment, with about $33 \%$ of the patients experiencing no pain, 59\% having mild pain, $7 \%$ having moderate pain, and only $1 \%$ having severe pain.

Of the 151 patients, $106(70.2 \%)$ presented with pathological fractures; $52.7 \%$ had long bone fractures, $44.5 \%$ had axial bone fractures, and $2.8 \%$ of patients had both axial and long bone fractures at the time of presentation. When these fractures were evaluated using the Mirels scoring system, ${ }^{(4)} 88.7 \%$ of the 
patients who presented with long bone fractures had a Mirels score $\geq 8$ and only $11.3 \%$ scored $\leq 7$. Only $15.4 \%$ of patients who presented without any pathological fracture had a Mirels score $\geq 8$. The majority of patients $(84.6 \%$ ) who presented without long bone pathological fractures scored $\leq 7$ (Table II).

Neurological deficit was assessed with the Frankel grading system. ${ }^{(6)}$ Among patients who presented with axial bone metastasis alone, or together with long bone metastasis, $90.7 \%$ (78/86) had some degree of neurological deficit at the time of presentation. Of these 86 patients, $4(4.7 \%)$ were classified as Frankel B, 30 (34.9\%) as Frankel C, 44 (51.2\%) as Frankel D, and $8(9.3 \%)$ as Frankel E. The neurological status of all 86 patients improved after treatment - Frankel A: 0\%, Frankel B: 1.2\%, Frankel C: $20.9 \%$, Frankel D: $50.0 \%$ and Frankel E: $27.9 \%$. (Table III).

The functional status of patients was assessed using the Brice and MacKissock grading scale. ${ }^{(4)}$ At presentation, $14.6 \%$ of patients had normal functional status (grade 1), $26.5 \%$ were able to ambulate with support (grade 2), 58.9\% were able to sit up only (grade 3), and none was completely bedridden (grade 4). Post treatment, there was significant improvement in the functional status of the patients - grade 1: $34.4 \%$; grade 2: $45.7 \%$; grade 3: $19.9 \%$ and grade $4: 0 \%$ (Table IV).

Of the 151 patients, $62(41.1 \%)$ had visceral metastasis and $89(58.9 \%)$ had metastasis confined to the skeletal system. Most of the bone lesions observed were osteolytic $(67.8 \%)$ in nature, while the rest were either sclerotic $(24.3 \%)$, or mixed osteolytic and osteosclerotic (7.9\%).

Patients with primary breast cancer had the longest mean survival time (21.0 months), followed by those with thyroid (20.7 months) and prostate (20.3 months) cancers. Patients with primary lung cancer had the shortest mean survival time (16.0 months). The mean survival time of patients with unknown primary cancer was 32.6 months, while the cumulative mean survival time of all the other cancers was 28.4 months (Table V). The Kaplan-Meier survival curve of our study cohort is shown in Fig. 2. The end point of survival was taken to be death.

The mean survival time of male patients in our cohort was 27.9 months, while that for female patients was 21.0 months $(p=0.03)$. The mean survival time was 24.8 months for patients with long bone skeletal metastases, 27.7 months for patients with axial bone skeletal metastases, and 18.4 months for patients with both long and axial bone skeletal metastases - the last group of patients had a statistically shorter mean survival time than the first two groups (Fig. 3).

The survival times of patients with respect to pathological fractures were as follows: no pathological fracture - 28.6 months; long bone pathological fracture - 20.9 months; axial bone pathological fracture - 26.4 months; both long and axial bone fractures - 13 months. There was a statistically significant difference in the mean survival times of patients who presented without any pathological fracture and those who presented with long bone pathological fractures (Fig. 4). Patients with extraskeletal metastases survived for an average of 24 months, whereas patients without extraskeletal metastases survived for an
Table II. Mirels scores of patients with no pathological fractures compared with those with long bone pathological fractures alone, or both axial and long bone pathological fractures.

\begin{tabular}{lccc}
\hline \multirow{2}{*}{$\begin{array}{l}\text { Mirels } \\
\text { score }^{(4)}\end{array}$} & \multicolumn{3}{c}{ No. of patients } \\
\cline { 2 - 4 } & None & Long bone & Axial and long bone \\
\hline$\leq 7$ & 22 & 6 & 0 \\
$\geq 8$ & 4 & 47 & 3 \\
Total & 26 & 53 & 3 \\
\hline
\end{tabular}

Table III. Pre- and post-treatment Frankel grades of patients with axial bone metastasis alone, or together with long bone metastasis $(n=86)$.

\begin{tabular}{lllllll}
\hline No. of patients & \multicolumn{4}{c}{ Post-treatment Frankel grade } & \multirow{2}{*}{ Total } \\
\cline { 2 - 5 } & A & B & C & D & E & \\
\hline
\end{tabular}

\begin{tabular}{|c|c|c|c|c|c|c|}
\hline \multicolumn{7}{|c|}{$\begin{array}{l}\text { Frankel grade } \\
\text { at presentation }\end{array}$} \\
\hline A & 0 & 0 & 0 & 0 & 0 & 0 \\
\hline$B$ & 0 & 1 & 3 & 0 & 0 & 4 \\
\hline C & 0 & 0 & 15 & 12 & 3 & 30 \\
\hline D & 0 & 0 & 0 & 31 & 13 & 44 \\
\hline$E$ & 0 & 0 & 0 & 0 & 8 & 8 \\
\hline Total & 0 & 1 & 18 & 43 & 24 & 86 \\
\hline
\end{tabular}

Note: Frankel grade A: complete motor and sensory paralysis; grade B: complete motor paralysis with some sensory preservation; grade C: retained motor function, but useless; grade D: retained useful motor function; grade E: free of neurologic symptoms

Table IV. Pre- and post-treatment Brice and MacKissock grades of the patients $(n=151)$.

\begin{tabular}{lcccc}
\hline No. of patients & \multicolumn{3}{c}{$\begin{array}{c}\text { Post-treatment Brice and } \\
\text { MacKissock grade }\end{array}$} & Total \\
\cline { 2 - 4 } & $\mathbf{1}$ & $\mathbf{2}$ & $\mathbf{3}$ & \\
\hline $\begin{array}{l}\text { Brice and MacKissock } \\
\text { grade at presentation }\end{array}$ & & & & \\
1 & 21 & 1 & 0 & 22 \\
2 & 10 & 30 & 0 & 40 \\
3 & 21 & 38 & 30 & 89 \\
Total & 52 & 69 & 30 & 151 \\
\hline
\end{tabular}

Note: Brice and MacKissock's grade 1: able to ambulate without support; grade 2: able to ambulate with support; grade 3 : able to sit up only

Table V. Mean survival time according to primary cancers $(n=151)$.

\begin{tabular}{lcc}
\hline Type of primary cancer & Survival time* & $\mathbf{9 5 \%} \mathbf{~ C l}$ \\
\hline Breast & $21.0 \pm 2.3$ & $16.5-25.6$ \\
Thyroid & $20.7 \pm 4.0$ & $12.8-28.6$ \\
Prostate & $20.3 \pm 2.3$ & $15.7-24.8$ \\
Lung & $16.0 \pm 1.7$ & $12.6-19.4$ \\
Unknown & $32.6 \pm 3.2$ & $26.2-38.9$ \\
Others & $28.4 \pm 1.9$ & $24.6-32.3$ \\
Overall & $24.7 \pm 1.3$ & $22.1-27.3$ \\
\hline
\end{tabular}

*Data is presented as mean \pm standard error. $\mathrm{Cl}$ : confidence interval

average of 31 months. This difference was statistically significant ( $p=0.001$, Fig. 5). On Cox regression analysis of all the risk factors studied, only the presence of extraskeletal metastasis $(p=0.05)$ and type of primary cancer $(p=0.034)$ were found to be significant. Factors such as gender $(p=0.421)$, site of metastasis, i.e. axial vs. long bone $(p=0.771)$, and the presence of pathological fractures $(p=0.838)$ were not significant. 


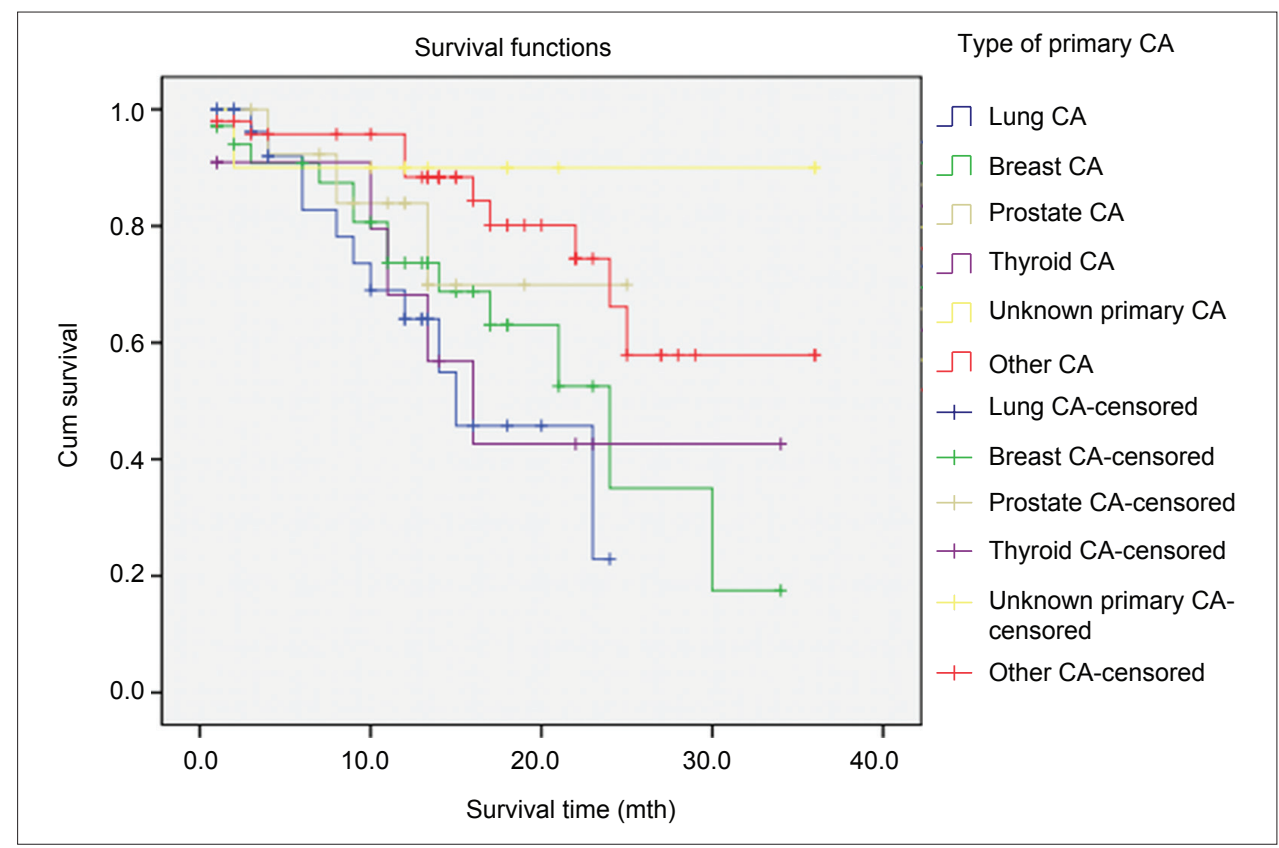

Fig. 2 Kaplan-Meier survival curve for the various cancers with bone metastasis. CA: cancer

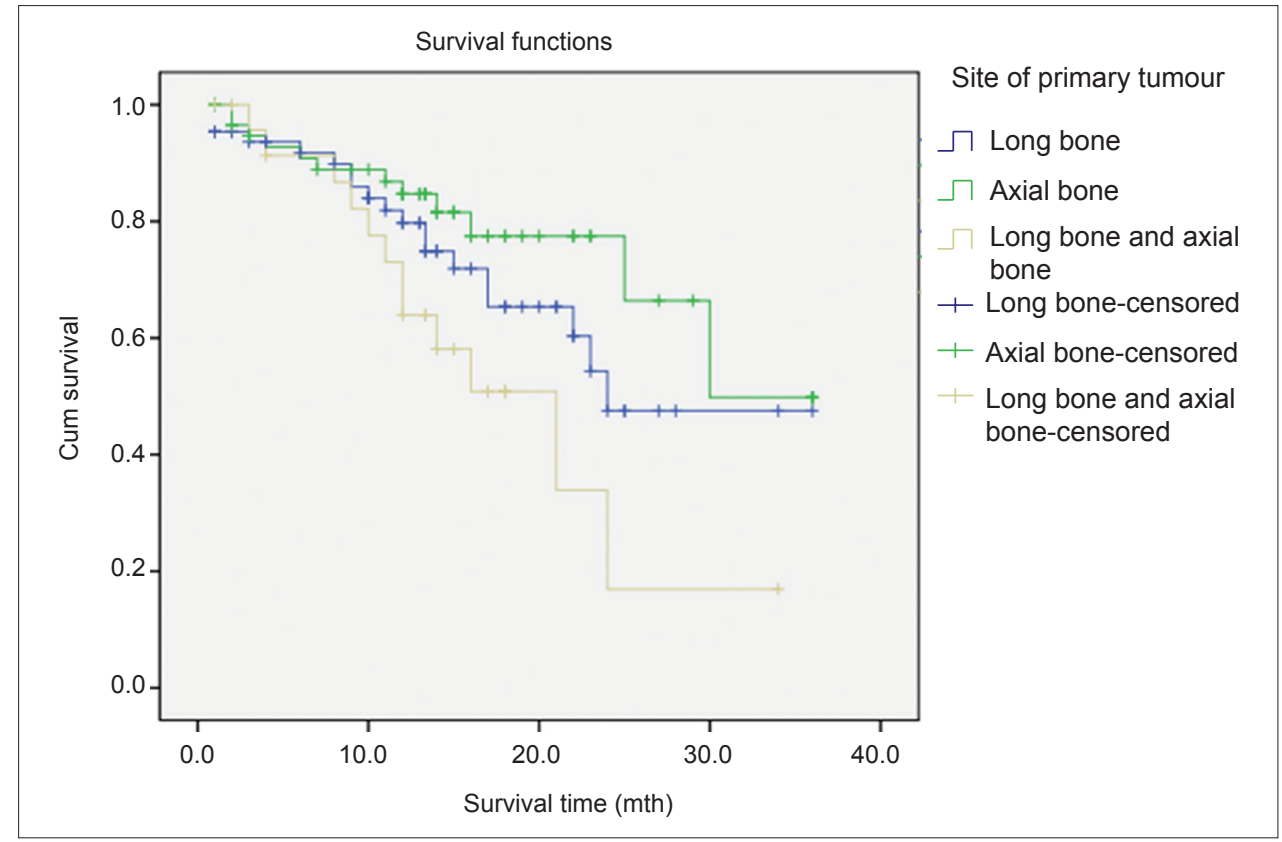

Fig. 3 Kaplan-Meier survival curves for the different sites of bone metastasis.

\section{DISCUSSION}

Over the years, the number of patients who present with metastatic bone disease has been increasing. This could be attributed to longer duration of survival due to advancements in cancer treatment, greater awareness among patients and better detection of metastatic disease. The skeletal system is the most commonly affected organ in metastatic disease, and it is also known to be the site of metastasis that incurs the highest morbidity. ${ }^{(7)}$

This study included 151 eligible patients who were diagnosed with metastatic bone disease from 1 January 2004 to 31 October 2009. All cases were retrospectively reviewed and the outcomes analysed. The gender distribution in our study population was almost equal (51\% male, 49\% female; ratio 1:1). Among female patients, breast cancer was the most prevalent primary cancer, while that of male patients were lung and prostate cancers. The age distribution of our cohort was largely based on the type of primary tumours of the patient. The highest incidence of metastatic bone disease was observed in patients aged $50-59$ years, followed by patients aged $60-79$ years. Even though most of our patients were of older age, one patient was only 18 years old. Therefore, metastatic bone disease should still be considered in the differential diagnosis even among younger patients who present with bone lesions.

Bone metastasis tends to occur more frequently in certain primary cancers. Coleman showed that close to $80 \%$ of 


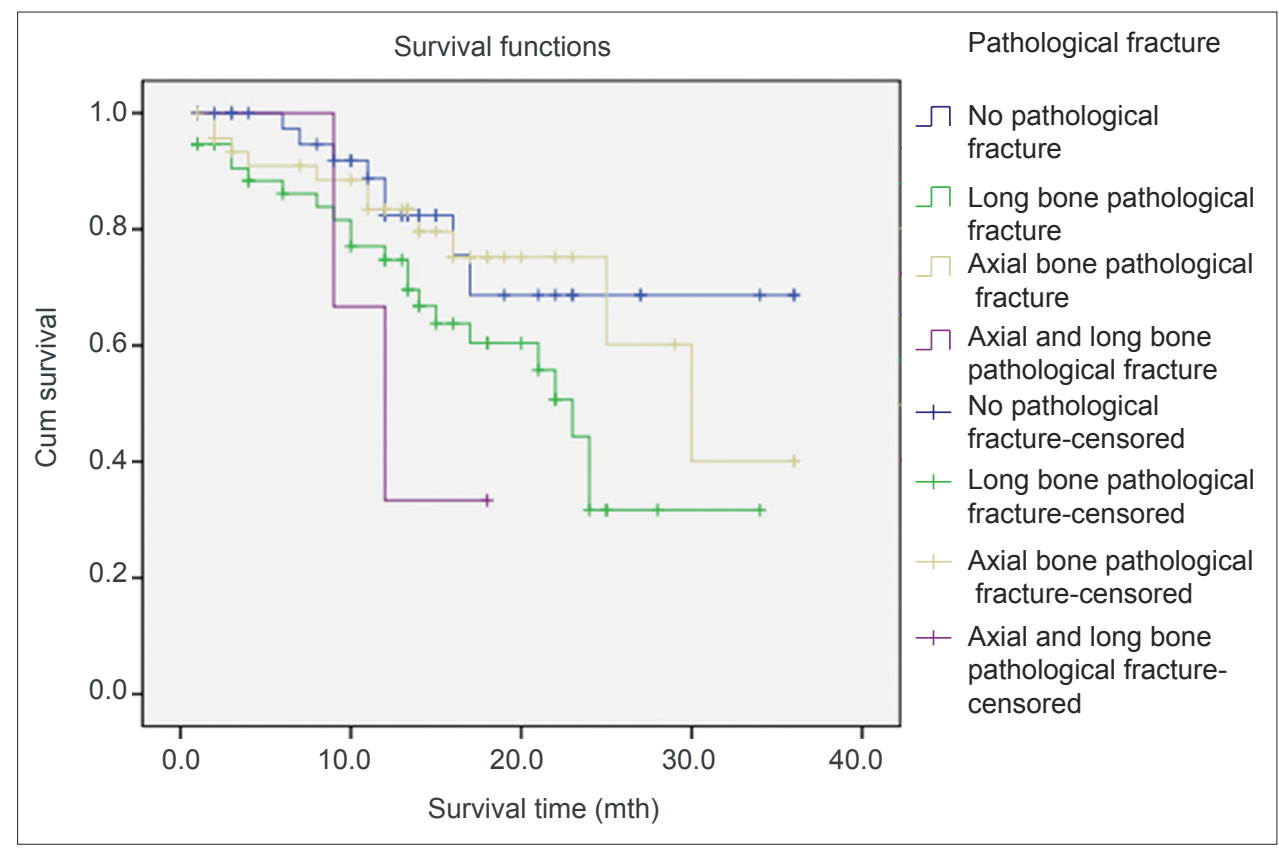

Fig. 4 Kaplan-Meier survival curves for patients with and without pathological fractures.

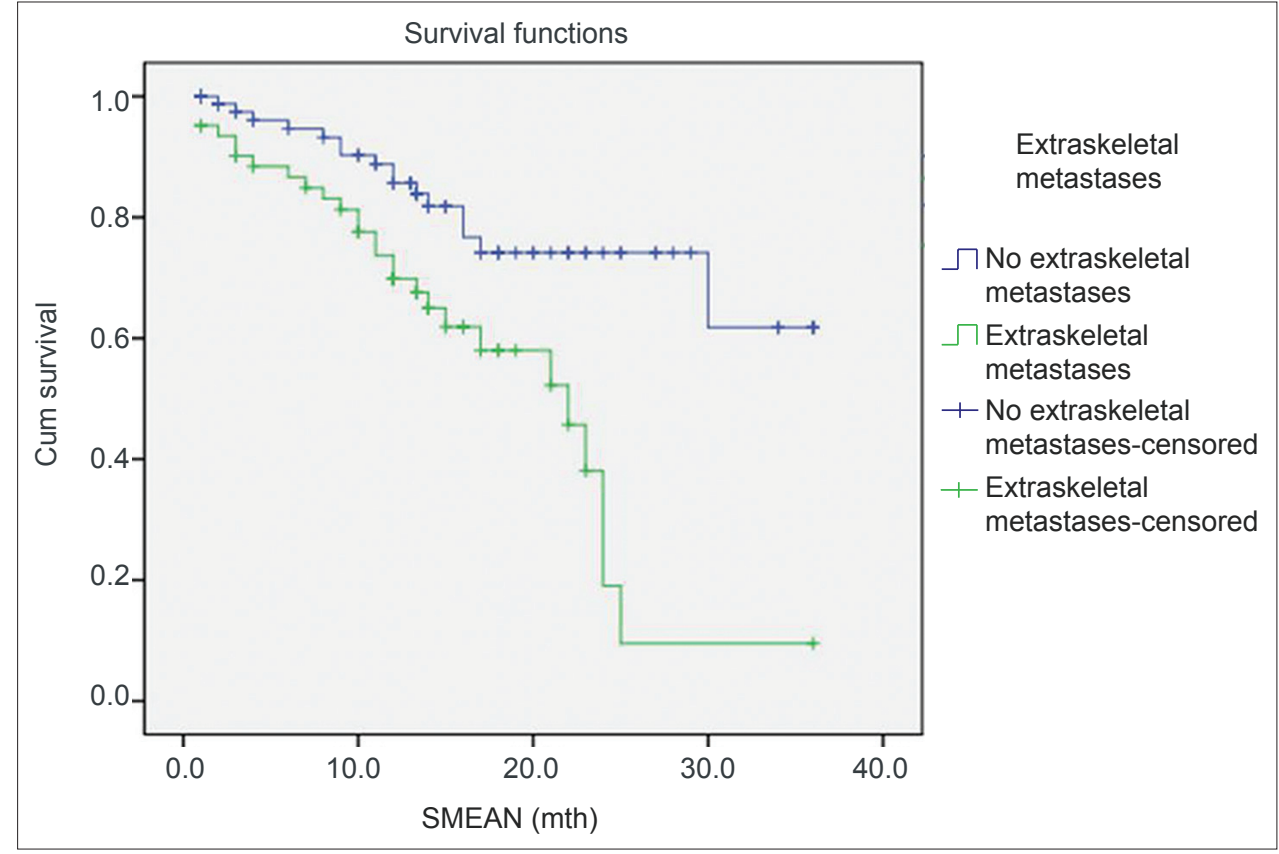

Fig. 5 Survival curves based on the presence of extraskeletal metastasis. SMEAN: mean survival time

advanced breast and prostate cancer patients would eventually develop skeletal metastases. ${ }^{(1)}$ Similarly, a postmortem study by Harrington ${ }^{(8)}$ reported high incidences of bone metastasis in breast and prostate cancers ( $84 \%$ ) and much lower incidences in other primary cancers such thyroid (50\%), lung $(44 \%)$ and renal (37\%) cancers. ${ }^{(8)}$ This could be due to the slow progression of breast and prostate cancers, allowing greater opportunity for the development of metastasis.

It has been reported that breast, lung and prostate cancers commonly metastasise to the spine, as they have a higher prevalence and predilection toward bone metastasis. ${ }^{(7)}$ In our cohort, there was a lower percentage of bone metastasis for prostate cancer compared to breast and lung cancers. This could be due to the low number of patients with prostate cancer referred to our unit. In our study, the primary cancers with the lowest incidence of metastatic bone disease were carcinoma of the larynx, ovarian cancer, oesophageal cancer, tongue cancer, squamous cell carcinoma, parathyroid cancer, endometrial cancer and malignant melanoma; only one case of metastatic bone disease was found in each of these primary cancers. Other primary cancers included hepatocellular carcinoma, lymphoma, testicular cancer, colorectal cancer, basal cell carcinoma and anaplastic meningioma.

Metastasis is more prevalent in the axial skeleton compared to the appendicular skeleton, as the former has a higher percentage of red bone marrow. The areas commonly involved are the ribs, 
pelvis and spine. This is partly due to Batson's vertebral venous plexus, which bypasses the lung circulation. ${ }^{(1,7)} \mathrm{A}$ cadaver study by Sundaresan et al has demonstrated that $30 \%-90 \%$ of patients with terminal cancer had spinal metastasis. ${ }^{(9)}$ In the present study, there was a marginally higher incidence of long bone metastasis than axial skeleton metastasis. This could be due to the fact that, in our centre, patients with extensive axial skeletal metastasis are not deemed suitable for surgical intervention, and are thus directly referred to the palliative team.

In general, two types of pain are experienced by patients with metastatic bone disease. The first is an ongoing pain that is dull and aching, or throbbing in nature; the severity of this type of pain increases over time. The second is a movement-evoked breakthrough or episodic pain, which is more acute in nature and often presents as spontaneous and intermittent exacerbations of pain or pain that is initiated by movement of the affected bone. ${ }^{(10)}$ The origin of pain associated with bone metastasis can be biological or mechanical. ${ }^{(1)}$ Biological pain is caused by the local release of cytokines and chemical mediators by tumour cells, periosteal irritation, or stimulation of the intraosseous nerves. Mechanical pain is due to pressure or mass effect of the tumour tissue within the bone, with the weakening of bone strength giving rise to activity-related pain. Although mechanical pain is common in osteolytic lesions, it is also present in osteoblastic lesions. This is due to the structural compromise of the affected bone. ${ }^{(11)}$

Pain is the principal symptom in patients who present with metastasis, ${ }^{(1)}$ and metastasis pain is the commonest symptom in metastatic bone disease, necessitating treatment. ${ }^{(12)}$ Pain is experienced by $30 \%-50 \%$ of all cancer patients, and pain that is life-modifying is experienced by $75 \%-90 \%$ of patients. ${ }^{(10,13,14}$ Bone pain caused by cancer is a challenge to treat. ${ }^{(15-17)}$ Tillman et al reported a $70 \%$ incidence of pain in patients with bone metastasis, describing it as dull in character and constant at night. ${ }^{(18)}$ In the present study, the majority of our patients $(90.1 \%)$ presented with pain as the primary symptom, consistent with the findings of most previous studies.

However, we opine that if metastatic bone disease is detected early and treated well, pain and other skeletal complications can be reduced, improving the patient's quality of life. In our study, most of the patients who underwent surgical procedures for both therapeutic and palliative purposes had significant reduction in pain and other skeletal complications. Out of the 93 patients who underwent operative treatment, about $75 \%$ of patients saw a reduction in their pain score and $50 \%$ became pain-free after surgery.

In metastatic bone disease, the most severe disabilities are caused by long bone fractures and tumour extension into the spinal cord. Pathological fractures begin as stress fractures and progress to complete fractures, which can severely affect the quality of life of patients with advanced disease. Chow et al reported that $10 \%-20 \%$ of such patients will have fractures of the weight-bearing long bone, while $5 \%$ will have spinal cord or nerve-root compression. ${ }^{(12)}$ Therefore, if we are able to predict the potential sites of fracture in metastatic bone disease, prophylactic surgery can be carried out to overcome any potential complications.
Mirels developed a scoring system for diagnosing impending pathologic fractures based on the site, nature and size of lesion, and symptoms; ${ }^{(4)}$ a score $>7$ indicates that surgical intervention is recommended and a score $>10$ indicates a fracture risk of more than $50 \% .{ }^{(18)}$ In 1991 , a study by Nielsen et $\mathrm{al}^{(2)}$ showed that $10 \%-30 \%$ of their cancer patients developed pathological fractures, mostly with involvement of the proximal parts of the long bones, especially the femur. If solid tumours were involved, the majority of all pathologic fractures occurred in patients with breast cancer $(60 \%)$, with only $10 \%$ occurring in patients with lung cancer. Major complications were encountered in one-third of these patients, $17 \%$ of whom required orthopaedic intervention. Of these $17 \%$, two-thirds of the cases involved femoral metastases. ${ }^{(2)}$ A study by Kunisada et al ${ }^{(19)}$ on 40 patients with periacetabular metastasis reported that the proximal femur was the most common site of pathological fracture requiring surgery. The authors recommended surgical treatment except when the surgical risks outweighed the benefits (e.g. surgery is not recommended for patients with a life expectancy of less than six weeks, and in bedridden patients). ${ }^{(19)}$

In the present study, our results concurred with the recommendations of Mirels. The higher rate of axial and long bone pathological fractures in our cohort (as compared to other studies) could be due to the fact that only patients who required surgical intervention were referred to our orthopaedic oncology unit. Surgical intervention is usually performed for the following indications: (a) prophylactic fixation of the involved bone when there is a fracture risk; (b) pathological fracture stabilisation; and (c) spinal decompression with or without stabilisation, if there is neurological deficit.

Neurological deficit is commonly present in cancer patients with bone metastasis. Spinal cord compression, for instance, is a medical emergency that requires urgent attention. Patients with spinal cord compression usually present with weakness, paralysis and sensory impairment. Late manifestation of spinal cord compression typically presents as urinary retention, incontinence and impotence. Lesions involving the conus medullaris can result in early autonomic dysfunction of the bladder, rectum and genitalia. In Posner et al's study, motor dysfunction was found to be the second most common presentation of patients with vertebral metastasis, with $60 \%-85 \%$ of patients showing some motor weakness. ${ }^{(20)}$

Early diagnosis, before the development of neurological deficit, is therefore essential, as it is correlated with better prognosis, especially in patients who present with spinal cord compression secondary to vertebral collapse. In a retrospective analysis of breast cancer patients with spinal cord compression $(96 \%$ had motor weakness, $94 \%$ had pain, $79 \%$ had sensory disturbance, $61 \%$ had sphincter disturbance), Schaberg and Gainor found that $91 \%$ of patients with spinal metastasis had at least one symptom for more than a week and $96 \%$ of those who were ambulant prior to treatment maintained their ability to ambulate after treatment. ${ }^{(3)}$

In all, 51.7\% (78/151) of our patients had some neurological deficit at presentation and about $40 \%$ had poor motor function at presentation. The neurological status of our patients 
improved significantly after treatment. Of the 92 patients who underwent surgical treatment, 78 had neurological compromise before surgery. Among these patients, 42.3\% (33/78) saw an improvement in their neurological status by at least one Frankel grade. The number of patients who had no useful motor function (i.e. Frankel B and C) at presentation was reduced from 34 to 19 after treatment. The number of patients who became free of neurological symptoms after treatment also improved from 8 to 24 patients. In our cohort, only $14.6 \%$ of the patients had normal functional status at presentation, while $26.5 \%$ were able to ambulate with support and $58.9 \%$ were only able to sit up. Post treatment, we likewise found considerable improvement in the functional status of our patients.

On radiography, metastatic bone disease can appear osteolytic, osteoblastic, or both. While a majority of spinal metastatic lesions are osteolytic, up to $50 \%$ of the bone must be eroded before a conspicuous change can be noted on plain radiographs. Osteoblastic or osteosclerotic lesions are commonly seen in carcinomas of the prostate and breast. ${ }^{(21)}$ Pathological fractures are commonly seen in patients with breast cancer due to the mainly lytic nature of the lesions. They are, however, infrequently seen in patients with lung and prostate cancers, as the survival rate is short in the former and the lesions are mainly osteoblastic in the latter. On plain radiographs, lytic lesions must be at least $1 \mathrm{~cm}$ across and affecting 30\%-50\% of bone density to be clinically detectable. ${ }^{(4)}$

Breast and lung cancers usually present with osteolytic lesions, while prostate cancers frequently present with sclerotic lesions. The majority (67.1\%) of our patients had lesions that were osteolytic in nature. Sclerotic lesions accounted for $24.3 \%$ of the observed lesions, while $7.9 \%$ of the lesions were a mixture of osteolytic and sclerotic densities. These findings coincide with the high incidence of breast and lung cancers, and the low incidence of prostate cancer among our patients.

Metastasis is now considered a chronic disease, and with the advent of new modalities of treatment, the lifespan of patients with metastatic disease has also improved. The survival of patients with metastatic disease is generally dependent on the type of primary tumour. For instance, patients with primary breast or prostate cancer may survive for years, while patients with primary lung cancer usually have shorter survival. In an Asian study, Tatsui et al ${ }^{(22)}$ reviewed the survivability of 425 patients with spinal metastasis detected by scintigraphy. The survival rate at one year was $83.3 \%$ for prostatic cancer, $77.7 \%$ for breast cancer, $51.2 \%$ for renal cancer, $44.6 \%$ for uterine cervical cancer, and $21.7 \%$ for lung cancer. The major prognostic factors were visceral metastases and grade of primary malignancy. ${ }^{(22)}$

In the present study, the mean survival time for lung cancer was 16 months, which is better than that reported in other studies - Tsuya et al: 6.2-12.2 months; ${ }^{(23)}$ Coleman: 6-9 months. ${ }^{(1)}$ This improvement in survival time may be attributed to the advancements in chemotherapy treatment. For breast cancer, the mean survival time in the present study was 21 months. This is in keeping with those published in other studies - Paterson et al: 2-3 years; ${ }^{(24)}$ Koenders et al: 34 months; ${ }^{(25)}$
Coleman: 19-25 months. ${ }^{(1)}$ The mean survival time for prostate cancer was 20.3 months in the present study, which is comparable to Coleman et al's findings (median 12-53 months), (1) but differs from Saad et al's findings (median 2-3 years depending on hormone responsiveness). ${ }^{(26)}$ For thyroid cancer, the present study showed a mean survival time of 20.7 months. In a review of 146 patients with thyroid cancer and bone metastasis, Pittas et al reported an overall survival of ten years in $13 \%-21 \%$ of patients, ${ }^{(27)}$ while Coleman reported a survival period of 48 months. ${ }^{(1)}$ As the aforementioned findings show, the longer duration of survival among cancer patients could have given rise to a higher incidence of skeletal complications.

Unknown primary cancer, defined as histologically confirmed metastatic carcinoma that occurs in the absence of a detectable primary cancer, ${ }^{(28,29)}$ is one of the ten most common cancers, accounting for $3 \%-5 \%$ of all malignancies in humans. ${ }^{(28)}$ It is the seventh to eighth most common cancer worldwide and the fourth most common cause of cancer-related death. ${ }^{(28)}$ In a retrospective study, Shaw et $\mathrm{al}^{(30)}$ reviewed all patients with a diagnosis of unknown primary cancer in a single cancer centre over 12 months. Their patients were grouped based on performance status and the presence or absence of liver metastases; patients with a performance status of 0 or 1 and no liver metastases survived the longest (15 months), whereas those with liver metastasis survived for only three months. ${ }^{(30)}$ In the present study, the high mean survival time of unknown primary cancer (i.e. 32.6 months), which is almost double that of the other studies, could be attributed to the small sample size.

In the present study, the mean survival time of other primary cancers was 28.4 months. Other primary cancers, which made up $27.1 \%$ of all primary cancers, included carcinoma of the larynx, hepatocellular carcinoma, lymphoma, ovarian cancer, testicular cancer, oesophageal cancer, colorectal cancer, tongue cancer, parathyroid cancer, squamous cell carcinoma, multiple myeloma, basal cell carcinoma, anaplastic meningioma, endometrial cancer, nasopharyngeal carcinoma, cervical carcinoma and malignant melanoma.

The presence or absence of visceral metastases to vital organs is critical for the prognostication of patients with bone metastases, a disease that is often considered a general condition. Several studies have reported that patients with visceral metastases to vital organs have worse prognoses than patients without visceral metastases to vital organs. ${ }^{(1,31,32)}$ In Coleman's study, which involved a review of 367 patients with breast cancer and bone metastasis, the median survival time of patients with extraskeletal metastasis was 1.6 years, while that of patients with only bone metastasis was 2.1 years. ${ }^{(7)}$ In Kuru et al's retrospective study involving 470 patients, $17 \%$ of patients had solitary skeletal metastasis, $22 \%$ had multiple skeletal metastases, and $61 \%$ had visceral metastasis. The group with solitary bone metastasis had the best survival at five years (73\%) followed by the groups with multiple bone metastases (46\%) and visceral metastasis (22\%). ${ }^{(33)}$ The results of Swenerton et al's study ${ }^{(34)}$ concur with those of Kuru et al's. Similarly, our findings confirm the relationship between extraskeletal metastases and short-term survival (mean 
survival time: without skeletal metastases 28.8 months; with skeletal metastases 18.6 months).

It has been suggested that the rate of skeletal complications in bone metastasis may be related to the level of bone resorption. In Brown et al's study, ${ }^{(35)}$ the bone resorption markers of 121 patients with metastatic bone disease who were receiving regular bisphosphonates were measured monthly; the level of bone resorption was found to be strongly correlated with the number of skeletal-related events and/or deaths. ${ }^{(35)}$

In terms of the mean survival time of bone metastasis patients with or without skeletal-related events, our study found that patients without pathological fractures (28.6 months) had a longer mean survival time than those with pathological fractures (long bone 20.9 months; axial bone 26.4 months). Likewise, Tsuya et al's Asian study of 259 patients with non-small cell lung cancer in stages III and IV found that patients with skeletalrelated events (pathological fractures, spinal cord compression, hypercalcaemia and palliative radiation therapy) had shorter survival times than those without skeletal-related events (mean survival time: 12.2 vs. 6.2 months). ${ }^{(23)}$

In conclusion, the present study showed that the presence of extraskeletal metastasis was the most important predictor of survival in our study cohort, followed by the type of primary cancer. Factors such as gender, site of the metastasis and presence of pathological fractures were not significant. With the ongoing advancements in cancer treatment, patients with metastatic bone disease are surviving longer, thus making them more susceptible to bone-related complications. Surgical treatment for both curative and palliative purposes can contribute to an improved quality of life in these patients. However, the survival time of these patients largely depends on the type of primary cancer and whether systemic metastasis is present.

\section{REFERENCES}

1. Coleman RE. Metastatic bone disease: clinical features, pathophysiology and treatment strategies. Cancer Treat Rev 2001; 27:165-76.

2. Nielsen OS, Munro AJ, Tannock IF. Bone metastases: pathophysiology and management policy. J Clin Oncol 1991; 9:509-24.

3. Schaberg J, Gainor BJ. A profile of metastatic carcinoma of the spine. Spine (Phila Pa 1976) 1985; 10:19-20.

4. Mirels H. Metastatic disease in long bones. A proposed scoring system for diagnosingimpending pathologic fractures. Clin Orthop Relat Res 1989; 256-64.

5. American Cancer Society. Cancer-related pain: A guide for patients and caregivers. http://www.cancer.org/acs/groups/content/documents/ document/acspc-030531.pdf. Accessed October 13, 2014.

6. Frankel HL, Hancock DO, Hyslop G, et al. The value of posturalreduction in the initial management of close injuries of the spine with paraplegia and tetraplegia. Paraplegia 1969; 7:179-92.

7. Coleman RE. Clinical features of metastatic bone disease and risk of skeletal morbidity. Clin Cancer Res 2006; 12:6243s-9s.

8. Harrington KD. Impending pathologic fractures from metastatic malignancy: evaluation and management. Instr Course Lect 1986; 35:357-81.

9. Sundaresan N, DiGiacinto GV, Krol G, Hughes JE. Spondylectomy for malignant tumors of the spine. J Clin Oncol 1989; 7:1485-91.
10. Mercadante S. Malignant bone pain: pathophysiology and treatment. Pain 1997; 69:1-18.

11. Selvaggi G, Scagliotti GV. Management of bone metastases in cancer: a review.Crit Rev Oncol Hematol 2005; 56:365-78.

12. Chow E, Hoskin P, van der Linden Y, Bottomley A, Velikova G. Quality of life and symptom end points in palliative bone metastases trials. Clin Oncol (R Coll Radiol) 2006; 18:67-9.

13. Mercadante $S$, Arcuri E. Breakthrough pain in cancer patients: pathophysiology and treatment. Cancer Treat Rev 1998; 24:425-32.

14. Portenoy RK, Lesage P. Management of cancer pain. Lancet 1999; 353:1695-700.

15. Banning A, Sjøgren P, Henriksen H. Pain causes in 200 patients referred to a multidisciplinary cancer pain clinic. Pain $1991 ; 45: 45-8$.

16. Coleman RE. How can we improve the treatment of bone metastases further? Curr Opin Oncol 1998; 10 Suppl 1:S7-13.

17. Coleman RE, Houston S, Purohit OP, et al. A randomised phase II study of oral pamidronate for the treatment of bone metastases from breast cancer. Eur J Cancer 1998; 34:820-4.

18. Tillman RM. The role of the orthopaedic surgeon in metastatic disease of the appendicular skeleton.Working Party on Metastatic Bone Disease in Breast Cancer in the UK. J Bone Joint Surg Br 1999; 81:1-2.

19. Kunisada T, Choong PF. Major reconstruction for periacetabular metastasis: early complications and outcome following surgical treatment in 40 hips. Acta Orthop Scand 2000; 71:585-90.

20. Posner JB, Howieson J, Cvitkovic E. "Disappearing" spinal cord compression: oncolytic effect of glucocorticoids (and other chemotherapeutic agents) on epidural metastases. Ann Neurol 1977; 2:409-13.

21. Gabriel K, Schiff D. Metastatic spinal cord compression by solid tumors. Semin Neurol 2004; 24:375-83.

22. Tatsui $H$, Onomura $T$, MorishitaS, Oketa $M$, Inoue $T$. Survival rates of patients with metastatic spinal cancer after scintigraphic detection of abnormal radioactive accumulation. Spine (Phila Pa 1976) 1996; 21:2143-8.

23. Tsuya A, Kurata T, Tamura K, Fukuoka M. Skeletal metastases in non-small cell lung cancer: a retrospective study. Lung Cancer 2007; 57:229-32.

24. Paterson AH, Powles TJ, Kanis JA, et al. Double-blind controlled trial of oral clodronate in patients with bone metastases from breast cancer. J Clin Oncol 1993; 11:59-65.

25. Koenders PG, Beex LV, Kloppenborg PW, Smals AG, Benraad TJ. Human breast cancer: survival from first metastasis. Breast Cancer Study Group. Breast Cancer Res Treat 1992; 21:173-80.

26. Saad F, Karakiewicz P, Perrotte P. The role of bisphosphonates in hormonerefractory prostate cancer. World J Urol 2005; 23:14-8.

27. Pittas AG, Adler M, Fazzari M, et al. Bone metastases from thyroid carcinoma: clinical characteristics and prognostic variables in one hundred forty-six patients. Thyroid 2000; 10:261-8.

28. Pavlidis N, Fizazi K. Cancer of unknown primary (CUP). Crit Rev Oncol Hematol 2005; 54:243-50

29. Krementz ET, Cerise EJ, Foster DS, Morgan LR Jr. Metastases of undetermined source. Curr Probl Cancer 1979; 4:4-37.

30. Shaw AT, Yeap BY, Mino-Kenudson M, et al. Clinical features and outcome of patients with non-small-cell lung cancer who harbor EML4-ALK. J Clin Oncol 2009; 27:4247-53.

31. Toyoda $Y$, Shinohara N, Harabayashi $T$, et al. Survival and prognostic classification of patients with metastatic renal cell carcinoma of bone. Eur Urol 2007; 52:163-8.

32. Tomita K, Kawahara N, Kobayashi T, et al. Surgical strategy for spinal metastases. Spine (Phila Pa 1976) 2001; 26:298-306.

33. Kuru B, Camlibel M, Dinc S, et al. Prognostic factors for survival in breast cancer patients who developed distant metastasis subsequent to definitive surgery. Singapore Med J 2008; 49:904-11.

34. Swenerton KD, Legha SS, Smith T, et al. Prognostic factors in metastatic breast cancer treated with combination chemotherapy. Cancer Res 1979; 39:1552-62.

35. Brown JE, Cook RJ, Major $\mathrm{P}$, et al. Bone turnover markers as predictors of skeletal complications in prostate cancer, lung cancer, and other solid tumors. J Natl Cancer Inst 2005; 97:59-69. 